Pacific Journal of Mathematics

MAPGERMS INFINITELY DETERMINED WITH RESPECT TO 


\title{
MAPGERMS INFINITELY DETERMINED WITH RESPECT TO RIGHT-LEFT EQUIVALENCE
}

\author{
LESLIE C. WILson
}

Mather has given both algebraic and geometric characterizations of finitely determined germs. We conjecture analogous characterizations of infinitely determined germs and prove parts of this conjecture. Recall that two mapgerms $f$ and $g$ are (right-left) equivalent if there are germs of diffeomorphisms $l$ and $r$ such that $f=l \circ g \circ r$. A mapgerm $f$ at $x$ is finitely determined if there is a $k$ such that every germ having the same $k$-jet as $f$ at $x$ is equivalent to $f ; f$ is infinitely determined if every germ having the same Taylor series at $x$ as $f$ is equivalent to $f$.

Let $E_{n}$ denote the space of germs at 0 in $R^{n}$ of $C^{\infty}$ real valued functions and let $m_{n}$ denote the unique maximal ideal in $E_{n}$. Let $E_{n}^{p}$ denote the set of $p$-tuples of elements of $E_{n}$; $m_{n}^{k}$ may denote $k$-tuples or may be the $k$ th power of $m_{n}$ which should be clear from context. If $f$ is analytic, let $f_{c}$ denote its complexification.

THEOREM 1.1. (Mather; see [2] and [4]). For $f$ in $m_{n}^{p}$, the following are equivalent:

(1) $f$ is finitely determined;

(2) $d f E_{n}^{n}+f^{*} E_{p}^{p} \supset m_{n}^{k} E_{n}^{p}$ for some $k>0$;

(3) (assuming $f$ analytic) $f_{c}$ is locally multistable in a deleted neighborhood of 0 .

Since $f_{C}$ is a germ, (3) is to be interpreted as saying that, for each representative $F$ of the germ $f_{c}$, there is a deleted neighborhood $U$ of 0 on which $F$ is locally multistable (i.e., for each finite $S \subset U$ the germ of $F$ at $S$ is stable).

Since $d f m_{n}^{n}+f^{*} m_{p}^{p}$ represents the tangent space to the orbit of $f$, the motivation for (2) is clear (except for the replacement of $m$ by $E)$. Also clear is that the analogous condition for infinite determination is gotten by replacing $k$ by $\infty$.

Gaffney (see [2]) explains the motivation for (3) as follows: "It is relatively easy to understand why a finitely determined germ should have this property. Any perturbation at zero, whose Taylor expansion vanishes to sufficiently high order there, can be removed by a coordinate change in source and target. However, by a suitable high order perturbation at zero, one can obtain any kind of a perturbation at some fixed $x$ different from zero. This low order perturbation at $x$ is also removed by the induced change in 
source and target at the origin. Thus, one would expect the germ at $x$ to be stable."

If the perturbation at zero is by polynomial terms, the effect is felt at $x$ whether complex or real. If the perturbation at zero is by flat functions, the quote still applies for real $x$. Thus we are led to:

(3a) (assuming $f$ is analytic) $f$ is locally multistable in a deleted neighborhood of 0 .

However, it is not known whether infinitely determined germs need be equivalent to analytic germs. Since there are flat functions which satisfy (3a)-aside from analyticity-we need to repace (3a) by a condition which guarantees that $f$ becomes unstable at a finite rate as we approach 0 .

We write $\boldsymbol{R}^{p r} \times J^{k}(n, p)^{r}$ for the space of $r$-tuples of $k$-jets of elements of $E_{n}^{p}$. If $f$ maps $U$ into $\boldsymbol{R}^{p}$, we define a map $\left(j^{k} f\right)^{r}$ into the above jet space in the obvious way. If the germ of $f$ at a finite set $S$ is unstable, then there is a subset of $p+1$ or fewer points on which it is also unstable. Further, its stability on this set depends only upon its $p+1$ jet. Thus, if $f$ is unstable at $\left\{x_{1}, \cdots, x_{r}\right\}$, we call $\left(j^{p+1} f\right)^{r}\left(x_{1}, \cdots, x_{r}\right)$ an unstable multijet, and let Uns denote the set of unstable multijets. If $f$ is unstable at 0 , then $\left(j^{p+1} f\right)^{r}$ is necessarily unstable at $D$, the set of $r$-tuples satisfying:

(a) at least one component is zero, or

(b) two or more component points are the same and are critical points of $f$.

Conjecture 1.2. For $f$ in $m_{n}^{p}$, the following are equivalent:

(1) $f$ is infinitely determined;

(2) $d f E_{n}^{n}+f^{*} E_{p}^{p} \supset m_{n}^{\infty} E_{n}^{p}$;

(3) $d\left(\left(j^{p+1} f\right)^{p+1}(x), U n s\right) \geqq C d(x, D)^{r}$, for some $C, r>0$.

Suppose $f$ is analytic and (3a) holds. Then $F=\left(j^{p+1} f\right)^{p+1}$ is analytic and $F^{-1}(U n s)=D$. We will show later that $U n s$ is the zero set of some analytic map $P$. Since $P \circ F$ is analytic, it satisfies a Lojasiewicz inequality. By Lemma 1.3 of [11], (3) holds. Of course, (3) implies (3a) even if $f$ isn't analytic.

In [11], we prove the corresponding conjectures to be true when left-right equivalence is replaced by $C, R, K$ or $L$ equivalence. We will henceforth assume the notation and terminology of that paper. The present conjecture is an order of magnitude more difficult than those proved in [11]. We prove the following partial results.

THEOREM 1.3. (1) implies (3). 
The proof, which is carried out in $\S 2$, is entirely different from that of "(1) implies (3)" in theorem 1.1, where powerful algebraic techniques are employed.

THEOREM 1.4. If $f$ is finitely K-determined, then (2) implies (1).

The proof is carried out in $\S 3$. The requirement that $f$ be finitely $K$-determined is a real restriction: $\left(x^{2}+y^{2}\right)^{2}$ is not finitely $K$-determined, but is $\infty-R$-determined, hence infinitely $A$-determined. Nevertheless, the restriction is not too great, as finitely determined germs are plentiful (see [10] for a discussion of this point).

THEOREM 1.5. If $f$ is analytic and finitely K-determined, then (3a) implies (2).

ExAmple 1.6. Let $f(x, y)=\left(x, y^{4}+x y^{2}\right)$. The critical set $C(f)$ is $2 y\left(2 y^{2}+x\right)=0 ; 0$ is the only nonfold point of $f$ in $C^{2}$. $f$ is twoto-one on $2 y^{2}+x=0$ and $d f$ has the same image at these double points, so $f$ is not stable on $\boldsymbol{R}^{2}-\{0\}$. Thus $f$ is not infinitely determined.

EXAMPLe 1.7. Let $f(x, y)=\left(x, y^{4}+x^{2} y^{2}\right) . \quad C(f)$ is $2 y\left(2 y^{2}+x^{2}\right)=0$ and 0 is the only nonfold point in $C^{2}$. Since $C(f) \cap \boldsymbol{R}^{2}$ is the $x$-axis, on which $f$ is an embedding, $f \mid\left(\boldsymbol{R}^{2}-\{0\}\right)$ is stable. Note that the points $\left(x, \pm x i / 2^{1 / 2}\right) \in C(f)$ have a common real image. The image of $d f$ at both these points is spanned by $e_{1}+2 x y^{2} e_{2}$ (where $e_{1}$ and $e_{2}$ form the standard basis). Thus $f_{c}$ is not stable on $f_{c}^{-1}\left(\boldsymbol{R}^{2}-\{0\}\right)$. In particular, $f$ is not finitely determined, but is infinitely determined.

Belickii, in [1], establishes a sufficient condition for a germ to be infinitely determined. However, his condition is very restrictiveit implies that, on a deleted neighborhood of 0 , the germ has only fold singularities and is an embedding on its fold set. There is also a mistake in the statement of his sufficient condition; for a discussion of this see [11]. (One should add that his principal result concerns germs of diffeomorphisms infinitely determined with respect to conjugation.) $\mathrm{He}$ also gives examples of infinitely determined germs.

There is a useful reformulation of condition (3). First we prove that $U n s$ is an algebraic variety. Given a mapgerm $f:\left(\boldsymbol{R}^{n}, S\right) \rightarrow \boldsymbol{R}^{p}$, define

$$
A:\left(\boldsymbol{R}^{n} \times J^{p}(n, n) \times \boldsymbol{R}^{p} \times J^{p}(p, p)\right)^{r} \longrightarrow \boldsymbol{R}^{p r} \times J^{p}(n, p)^{r}
$$

to be the real linear map induced by 


$$
\left(d f, f^{*}\right): E_{S}^{n} \times E_{f(S)}^{p} \longrightarrow E_{S}^{p} .
$$

$A$ depends only upon $z=\left(j^{p+1} f\right)^{r} \in \boldsymbol{R}^{p r} \times J^{p+1}(n, p)^{r}$ and is surjective iff $z$ is stable. Considered as a matrix, the coefficients of $A$ are polynomials on $\boldsymbol{R}^{p r} \times J^{p+1}(n, p)^{r}$. Let $I$ be the polynomial ideal generated by the determinants of the maximal minors of $A$. Then $U n s$ is the zero set of $I$.

Let $I(f)=\left(\left(\left(j^{p+1} f\right)^{p+1}\right)^{-1} I\right) E_{(p+1) n}$. Then condition (3) is equivalent to requiring that $I(f)$ be a Lojasiewicz ideal at $D$, which is equivalent to $I(f) \supset m_{D}^{\infty}$ (see V. 4.3 of [10]).

2. Proof of Theorem 1.3. We assume that $f$ does not satisfy condition (3). We will prove that two representatives of the Taylor series $T f$ of $f$ at 0 exist which are inequivalent.

We need a method to prove that two maps are inequivalent. If they are equivalent, their singularity sets of each type are diffeomorphic. The strategy then is to find one representative whose singularities of some type are very nice, and another whose singularities of that type are demonstrably bad.

The nice representative will be found using a consequence of the Multijet Transversal Extension Theorem (see [11]). First some terminology and notation. The generalized diagonal in $\boldsymbol{R}^{n r}$ is $\left\{\left(x_{1}, \cdots, x_{r}\right): x_{i} \neq x_{j}\right.$ for some $\left.i \neq j\right\}$. Let $\left(\boldsymbol{R}^{n}\right)^{(r)}$ denote $\boldsymbol{R}^{n r}$-(generalized diagonal) and let ${ }_{r} j^{k} f$ denote $\left(j^{k} f\right)^{r} \mid\left(\boldsymbol{R}^{n}\right)^{(r)}$. A deleted neighborhood of 0 in $\left(\boldsymbol{R}^{n}\right)^{(r)}$ means a neighborhood of 0 less those points, at least one component of which is 0 .

Lemma 2.1. Given a formal power series $z$ and a countable collection of submanifolds of $\boldsymbol{R}^{p r} \times J^{k}(n, p)^{r}$, there is a residual set of representatives $f$ of $z$ for which ${ }_{r} j^{k} f$ is transverse on a deleted neighborhood of 0 to each of the submanifolds.

We have two devices for constructing bad representatives. The first was proved in [11], and is used to construct a representative having singular jets along a given sequence.

Lemma 2.2. Suppose there exist $w_{i}$ in $\boldsymbol{R}^{p r} \times J^{k}(n, p)^{r}(k \leqq \infty)$, $x_{i}=\left(x_{i}^{1}, \cdots, x_{i}^{r}\right)$ in $\boldsymbol{R}^{n r}$ converging to 0 , and $f$ in $E_{n}^{p}$ such that $q_{i}=$ $w_{i}-\left(j^{k} f\right)^{r}\left(x_{i}\right)$ is flat along $\left|x_{i}^{s}\right|$ for each $s$ and along $\left|x_{i}^{s}-x_{i}^{t}\right|$ for each $s \neq t$. Then there is a $g$ such that $T g=T f$ and $\left(j^{k} g\right)^{r}\left(x_{i}\right)=w_{i}$ holds for a subsequence of $\left\{x_{i}\right\}$.

The second device is used to construct a representative which fails to be transverse to some given singularity. 
LEMma 2.3. Let $\left\{S_{j}\right\}$ be a collection of manifolds whose union is a closed set $S \subset J^{k}(n, p)$. Let $f$ be a $C^{\infty}$ map restricted to a closed disk $D$ centered at 0 . Assume $V=\left(j^{k} f\right)^{-1} S$ contains 0 . Suppose there is a sequence $x_{i}$ converging to 0 such that $d\left(j^{k} f\left(x_{i}\right), S\right)$ is flat along $d\left(x_{i}, V\right)$. Let $D_{i}$ be a closed disk centered at $x_{i}$ such that $V, D_{1}, D_{2}, \cdots$ are mutually disjoint. Then there is a $y_{i}$ in the open disk $D_{i}^{0}$ and a $g$ such that:

(i) $T f=T g$ and, on $D-\cup D_{i}^{0}, g=f$;

(ii) $j^{k} g\left(y_{i}\right) \in S_{j}$ for some $j$ and $j^{k} g$ is not transverse to $S_{j}$ at $y_{i}$.

Proof. By assumption, there exist $w_{i} \in J^{k}$, flat along $d\left(x_{i}, V\right)$, such that $j^{k} f\left(x_{2}\right)+w_{i} \in S$. Let $p_{i}$ be the polynomial of degree $k$ with $j^{k} p_{\imath}\left(x_{i}\right)=w_{i}$. For each $l \geqq k$, the $C^{l}$ norm $\left|p_{i}\right|_{D_{i}}^{l}=\left|p_{i}\right|_{D_{i}}^{k}$ is flat along $d\left(x_{i}, V\right)=d_{i}$.

Using Lemma IV. 3.3 of [10], there is, for each $i$, a $C^{\infty} a_{i}$ such that $a_{i}=1$ on a neighborhood of $x_{i}, a_{i}=0$ off $D_{i}^{0}$ and, for each multi-index $I$, a constant $C_{I}$ independent of $i$ such that

$$
\left|D^{I} a_{i}(x)\right| \leqq C_{I} / d_{i}^{|I|} \text {. }
$$

Thus, $\left|a_{i} p_{i}\right|_{D}^{l}$ is flat along $d_{i}$ for each $l$. Thus, for any sequence of numbers $t_{i}$ between 0 and $1, f+\sum t_{2} a_{i} p_{i}$ converges in the $C^{\infty}$ topology to some $C^{\infty}$ map $g$. This $g$ satisfies (i).

Fix an $i$. Let $g_{t}$ denote the map $g$ as defined above with $t_{i}=t$ (hold the other $t_{j}$ fixed). $j^{k} g_{t}(x)$ depends smoothly on both $x$ and $t$. Thus the set of $t$ such that $j^{k} g_{t}\left(D_{\imath}\right) \cap S=\varnothing$ is open and contains 0 . Hence there is an $s, 0<s \leqq 1$, and a $y_{i} \in D_{i}$ such that

(iii) $j^{k} g_{t}\left(D_{i}\right) \cap S=\varnothing$ for all $t<s$ and $j^{k} g_{s}\left(y_{i}\right) \in S_{j}$ for some $j$. However, by (i), $j^{k} g_{s}\left(D_{i}-D_{i}^{0}\right)$ misses $S$. Thus $y_{i}$ is in $D_{i}^{0}$. If $j^{k} g_{s}$ were transverse to $S_{j}$ at $y_{i}$, then for each $t$ near $s$ there would be some $x$ in $D_{i}^{0}$ with $j^{k} g_{t}(x) \in S_{j}$. But this contradicts (iii).

First we describe the stratification to which we'll apply Lemma 2.1. By a stratification, we mean a locally finite partition into embedded submanifolds. Let $A=\operatorname{Diff}\left(\boldsymbol{R}^{n}, 0\right) \times \operatorname{Diff}\left(\boldsymbol{R}^{p}\right)$ act on $\boldsymbol{R}^{p r} \times$ $J^{k}(n, p)^{r}$ in the usual way.

From Mather's results in [6], it follows that there is a stratification $\mathscr{S}$ of $\boldsymbol{R}^{p r} \times J^{k}(n, p)^{r}$ such that each stratum $S$ is $A$-invariant and semialgebraic, $S / A$ is an analytic manifold each compoent of which has the same dimension $c$, and $\pi: S \rightarrow S / A$ is analytic. If $c>0$ and $Q$ is an $A$-orbit in $S$, then a chart on $S / A$ is given by any sufficiently small direct transversal $L$ to $Q$ in $S$-in particular, each $A$-orbit sufficiently near to $Q$ in $S$ intersects $L$ exactly once. If $c>0$, we call $S$ an $A$-moduli stratum.

Assume $f$ does not satisfy condition (3). By Lemma 2.1, there 
is an $h$ with $T h=T f$ such that, on a deleted neighborhood of 0 , ${ }_{r} j^{p+1} h$ is transverse to the above-defined $\mathscr{S}$ for $r=1, \cdots, p+1$ and $j^{1} h$ is transverse to the stratification of $J^{1}(n, p)$ by rank.

Case $A . \quad h$ is not locally multistable on any deleted neighborhood of 0 .

In this case, there is a smallest $r$ between 1 and $p+1$ such that ${ }_{r} j^{p+1} h$ has unstable values on every deleted neighborhood of 0 . Suppose $z={ }_{r} j^{p+1} h(x)$ is in Uns. Since multitransversality to $A$ orbits is equivalent to stability (of a germ on a finite set), and $h$ is multitransverse to all $A$-orbits except those in the $A$-moduli strata, it follows that $z$ lies in an $A$-moduli stratum $S$. Let $x=\left(x_{1}, \cdots, x_{r}\right)$. By the minimality property of $r$, if $r$ is greater than 1 , then $h$ is stable at each $x_{i}$ (in fact at each proper subset of $\left\{x_{1}, \cdots, x_{r}\right\}$ ) and $h\left(x_{i}\right)$ has the same value for each $i$. It follows from a result in [12] that the $A$-orbit $Q$ of $z$ has codimension larger than $n r$ (when $r=1$, this is easy: an orbit is stable iff it has a transverse representative iff it has codimension $\leqq n)$. At any point $y$ such that ${ }_{r} j^{p+1} h(y) \in S,{ }_{r} j^{p+1} h$ is transverse at $y$ to $S$; for a sufficiently small neighborhood $U_{y}$ of $y,{ }_{r} j^{p+1} h\left(U_{y}\right) \cap S$ is a manifold of dimension less then the codimension of $Q$ in $S$. Thus $\pi\left(i m_{r} j^{p+1} h \cap S\right)$ is nowhere dense in $S / A$. Thus we can choose a $w$ in $S$ arbitrarily near $z$ which is inequivalent to every jet ${ }_{r} j^{p+1} h(y)$.

There is a sequence $x_{i}$ in a deleted neighborhood of 0 which converges to 0 such that $z_{i}={ }_{r} j^{p+1} h\left(x_{i}\right)$ is unstable. Then we can choose $w_{i}$ inequivalent to every jet ${ }_{r} j^{p+1} h(y)$ such that $w_{i}-z_{i}$ is flat along $\left|x_{i}^{s}\right|$ for all $s$ and along $\left|x_{i}^{s}-x_{i}^{t}\right|$ for every $s \neq t$. By Lemma 2.2 , there is a map $g$ such that $T g=T h$ and ${ }_{r} j^{p+1} g\left(x_{i}\right)=w_{i}$. Clearly $g$ is not equivalent to $h$.

If Case A doesn't hold, then $h$ is locally multistable on some deleted neighborhood of 0 . Since $f$ doesn't satisfy (3), there is a sequence $x_{i}$ converging to zero such that (letting $z_{i}=\left(j^{p+1} f\right)^{p+1}\left(x_{i}\right)$ ) $d\left(z_{i}, U n s\right)$ is flat along $d\left(x_{i}, D\right)$. This implies that $d\left(z_{i}, U n s\right)$ is flat along

$$
d_{i}=\min \left\{\left|x_{i}^{1}\right|, \cdots,\left|x_{i}^{p+1}\right|, e_{i}\right\}
$$

where $e_{i}$ is the minimum of $\left|x_{i}^{s}-c\right|+\left|x_{i}^{t}-c\right|$ over all $s \neq t$ and critical points $c$ of $f$.

Let $w_{i}$ be the nearest element of Uns to $z_{i}$. Form $w_{i}^{\prime} \in \boldsymbol{R}^{p r} \times$ $J^{p+1}(n, p)^{r}$ by omitting from $w_{i}$ all noncritical components. Form $x_{i}^{\prime}$ and $z_{i}^{\prime}$ by omitting from $x_{i}$ and $z_{i}$ the corresponding components. Define $d_{i}^{\prime}$ by omitting from $\left({ }^{*}\right)$ all the omitted components of $x_{i}$. From now on, we write $w_{i}$ for $w_{i}^{\prime}$, etc. 
Case B. $\left|z_{i}-w_{i}\right|$ is also flat along $\left|x_{i}^{s}-x_{i}^{t}\right|$, for all $s \neq t$.

Then, by Lemma 2.2, there is a $g$ such that $T g=T f$ and $\left(j^{p+1} g\right)^{r}\left(x_{i}\right)=w_{i}$. Thus $g$ and $h$ are inequivalent.

Case C. For some $s \neq t,\left|z_{i}-w_{i}\right|$ is not flat along $\left|x_{i}^{s}-x_{i}^{t}\right|$.

Then (passing to a subsequence of $x_{i}$ if necessary, and renumbering) $\left|x_{i}^{s}-x_{i}^{t}\right|$ is flat along $\left|x_{i}^{s}\right|,\left|x_{i}^{t}\right|$ and $\left|x_{i}^{s}-c_{i}\right|+\left|x_{i}^{t}-c_{i}\right|$, for some $c_{i} \in C(f)$. Then $\left|x_{i}^{s}-c_{i}\right|+\left|x_{i}^{t}-c_{i}\right|$ is approximately $2\left|x_{i}^{s}-c_{i}\right|$ for $i$ large. So $\left|z_{i}^{s}-w_{i}^{s}\right|$ is flat along $d\left(x_{i}^{s}, C(f)\right)$ and $w_{i}^{s}$ is a critical jet. By Lemma 2.3, there is a $g$ with $T g=T f$ such that $g$ is not transverse to the stratification of $J^{1}(n, p)$ by rank on any deleted neighborhood of 0 . Thus $g$ and $h$ are inequivalent.

3. Proof of Theorem 1.4. We are given that $f$ is finitely $K$ determined and

$$
d f E_{n}^{n}+f^{*} E_{p}^{p} \supset m_{n}^{\infty} E_{n}^{p} .
$$

Let $g(x, t)=f(x)+t u(x), u$ flat, let $F=(f, t)$ and $G=(g, t)$, and let $f^{a}, g^{a}, F^{a}$ and $G^{a}$ denote the germs of these maps at $(0, a)$ (where $f(x, t)=f(x)$ for all $t)$. Via translation we identify the germs at $(0, a)$ with those at $(0,0)$. Thus $g^{a}-f^{a}$ is in $m_{n}^{\infty} E_{n+1}^{p}$. We fix $a$ and henceforth will suppress the superscript $a$, writing $F$ for $F^{a}$, etc.

Let $m_{T}^{k}$ denote the germs which vanish to order $k$ along the $t$-axis in either $\boldsymbol{R}^{n+1}$ or $\boldsymbol{R}^{p+1}$. Then $m_{n}^{\infty} E_{n+1}=m_{T}^{\infty}$ and $m_{p}^{\infty} E_{p+1}=m_{T}^{\infty}$.

It is clear that $d(g-f) E_{n+1}^{n} \subset\left(m_{T}^{\infty}\right)^{p}$. If $V$ is in $E_{p+1}^{p}$, then $T(V \circ G-V \circ F)=0$ on the $t$-axis, so $V \circ G-V \circ F$ is in $\left(m_{T}^{\infty}\right)^{p}$.

We will later prove:

$$
d f E_{n+1}^{n}+F^{*} E_{p+1}^{p} \supset\left(m_{T}^{\infty}\right)^{p} .
$$

Assuming this, we have

$$
\begin{gathered}
d g E_{n+1}^{n}+G^{*} E_{p+1}^{p} \subseteq d f E_{n+1}^{n}+F^{*} E_{p+1}^{p} \\
=d g E_{n+1}^{n}+G^{*} E_{p+1}^{p}+\left(m_{\Gamma}^{\infty}\right)^{p} .
\end{gathered}
$$

Our goal is to prove equality in (3.3). It then follows that $\left(m_{T}^{\infty}\right)^{p} \subset d g E_{n+1}^{n}+G^{*} E_{p+1}^{p}$. Multiplying both sides by $G^{*} m_{T}$, we have

$$
\begin{aligned}
G^{*}\left(m_{T}\right)\left(m_{T}^{\infty}\right)^{p} & \subset d g G^{*} m_{T} E_{n+1}^{n}+G^{*}\left(m_{T}^{p}\right) \\
& \leqq d g m_{T}^{n}+G^{*}\left(m_{T}^{p}\right)
\end{aligned}
$$

Since $f$ is $\infty-K$-determined, it follows from the remark immediately following Lemma 2.1 of [11] that $\left(m_{T}^{\infty}\right)^{p} \subset d g\left(m_{T}^{\infty}\right)^{n}+G^{*}\left(m_{T}\right)\left(m_{T}^{\infty}\right)^{p}$. Thus, 


$$
\left(m_{T}^{\infty}\right)^{p} \subset d g m_{T}^{n}+G^{*}\left(m_{T}^{p}\right) .
$$

Then the argument of Mather in $\S 6.3$ of [4] shows that $g_{t}$ is equivalent to $g_{0}=f$ for all $t$, and the diffeomorphisms depend smoothly on $t$.

Proof of 3.2. Recall that we assumed $f$ is finitely $K$-determined. Choose $a_{1}, \cdots, a_{s}$ in $E_{n}$ whose projections into $E_{n} / J f+\left(f^{*} m_{p}\right) E_{n}$ form a real vector space basis. Since

$$
\left(F^{*} m_{p+1}\right) E_{n+1} \supset f^{*}\left(m_{p}\right) E_{n}+t E_{n+1},
$$

$E_{n+1} /(J f) E_{n+1}+\left(F^{*} m_{p+1}\right) E_{n+1}$ is also generated as a real vector space by the projections of $a_{1}, \cdots, a_{s}$. By the Malgrange Preparation Theorem, $E_{n+1} /(J f) E_{n+1}$ is generated by $a_{1}, \cdots, a_{s}$ as an $F^{*} E_{p+1}$ module. In particular,

$$
(J f) E_{n+1}+\left(F^{*} E_{p+1}\right) E_{n}=E_{n+1} \text {. }
$$

Since $d f E_{n+1}^{n} \supset J f E_{n+1}^{p}$ and

$$
\begin{aligned}
d f E_{n+1}^{n}+F^{*} E_{p+1}^{p} & \supset\left(d f E_{n}^{n}+F^{*} E_{p+1}^{p}\right) F^{*} E_{p+1} \\
& \supset\left(d f E_{n}^{n}+f^{*} E_{p}^{p}\right) F^{*} E_{p+1} \\
& \supset\left(m_{n}^{\infty}\right)^{p} F^{*} E_{p+1} \text { by }(3.1),
\end{aligned}
$$

it follows that

$$
\begin{aligned}
d f E_{n+1}^{n}+F^{*} E_{p+1}^{p} & \supset\left(m_{n}^{\infty}\right)^{p}\left(J f E_{n+1}+F^{*} E_{p+1} E_{n}\right) \\
& =\left(m_{n}^{\infty}\right)^{p} E_{n+1} \quad \text { by }(3.4) .
\end{aligned}
$$

Proof that equality holds in (3.3). Let $t G$ denote $d g E_{n+1}^{n}$ and $w G$ denote $G^{*} E_{p+1}^{p}$ (etc. for $F$ ). Then (3.3) says

$$
t G+w G \leqq t F+w F=t G+w G+\left(m_{T}^{\infty}\right)^{p} .
$$

Since $G$ is $\infty$ - $K$-determined,

$$
\begin{aligned}
\left(m_{\Gamma}^{\infty}\right)^{p} & =\left(t G+G^{*} m_{p+1} E_{n+1}^{p}\right) m_{T}^{\infty} \\
& \subset t G+G^{*} m_{p+1}(t F+w F) .
\end{aligned}
$$

By (3.5) and (3.6),

$$
(t G+w G)+G^{*} m_{p+1}(t F+w F)=t F+w F .
$$

Let $A=(t F+w F) / t G$. We will show: (3.7) $A$ is finitely generated as a $G^{*} E_{p+1}$ module. Then, by Nakayama's lemma,

$$
\begin{gathered}
(t G+w G) / t G=(t F+w F) / t G, \text { so } \\
t G+w G=t F+w F,
\end{gathered}
$$


which is what we want to prove.

To prove (3.7), it suffices by Gaffney's Preparation Theorem (see [2]) to show that (3.8) $A$ is f.g. as an $(F, G)^{*} E_{2 p+2}$ module, and (3.9) $A /\left(G^{*} m_{p+1}\right) A$ has finite real dimension.

By (3.5), $A /\left(G^{*} m_{p+1}\right) A$ is isomorphic to

$$
\left(t G+w G+\left(m_{T}^{\infty}\right)^{p}\right) /\left(t G+G^{*} m_{p+1}\left(t G+w G+\left(m_{T}^{\infty}\right)^{p}\right)\right) .
$$

Since the denominator contains $\left(G^{*} m_{p+1}\right)^{p}$ and $t G+\left(G^{*} m_{p+1}\right)\left(m_{T}^{\infty}\right)^{p}$, which contains $\left(m_{T}^{\infty}\right)^{p}$, the numerator is spanned by the denominator and the constant vector fields, proving (3.9).

Next we check that $(t F+w F) / t G$ is an $(F, G)^{*} E_{2 p+2}$ module. Pick $h$ in $E_{2 p+2}$. Then $l=h(F, G)-h(F, F)$ is in $m_{T}^{\infty}$. Since $t F+w F$ contains $\left(m_{T}^{\infty}\right)^{p}$,

$$
h(F, G)(t F+w F)=h(F, F)(t F+w F)+l(t F+w F) \subset t F+w F .
$$

$t G$ is an $E_{n+1}$ module, hence an $(F, G)^{*} E_{2 p+2}$ module, and is a submodule of $t F+w F$ by (3.5).

Finally, we must prove (3.8). $w F$ is f.g. over $F^{*} E_{p+1}$, hence over $(F, G)^{*} E_{2 p+2} . \quad t F$ is generated over $E_{n+1}$ by $\partial_{i}=\partial f / \partial x_{i}, i=$ $1, \cdots, n$. $t G$ contains $J G E_{n+1}^{p}$. Thus

$$
(t F+w F) / t G=\overline{w F}+\sum \bar{\partial}_{i}\left(E_{n+1} / J G\right),
$$

where the bar denotes the projection into the quotient space. Since we assume $f$ is finitely $K$-determined, so are $F$ and $G$. Thus $E_{n+1} / J G$ is finite as a $G^{*} E_{p+1}$ module by the Malgrange Preparation Theorem. Thus $A$ is finitely generated as an $(F, G)^{*} E_{2 p+2}$ module.

This completes the proof that equality holds in (3.3) and hence completes the proof of Theorem 1.4.

4. Proof of Theorem 1.5. The proof that "(3) implies (2)" in Theorem 1.1 depends essentially on the coherence theorem for pushforward sheaves. Merrien in [9] (summarized in [7] and [8]) has developed a notion of semicoherence which plays a role for real analytic maps and semianalytic sets analogous to that of coherence for complex analytic maps and varieties. The two theorems below were communicated to me by Merrien. They generalize Theorems II. 7.1 and IV. 7.3 of [9], and are proved in the same way. Let $\mathcal{O}$ and $\mathscr{E}$ be the sheaves of real analytic and $C^{\infty}$ functions on $\boldsymbol{R}^{n}$ (or $\boldsymbol{R}^{p}$; which will be clear from context). For any sheaf $\mathscr{M}$ let $\mathscr{M}_{U}$ denote its restriction to $U$ and $\mathscr{C}(U)$ the ring of sections into $\mathscr{M}$ over $U$. If $\mathscr{M}=\mathscr{E} p / \mathscr{F}$, then $\mathscr{M}(U)$ is given the quotient topology of the compact-open $C^{\infty}$ topologies, and $\widetilde{\mathscr{C}}(U)$ is, by definition, $\mathscr { E } ^ { p } ( U ) \longdiv { \mathscr { I } ( U ) }$. 
THEOREM 4.1. Let $f: U \rightarrow V, U \subset \boldsymbol{R}^{n}, V \subset \boldsymbol{R}^{p}$, be analytic and let $\mathscr{C}$ be an analytic semicoherent sheaf on $U$ such that

(i) $f \mid \operatorname{Supp} \mathscr{A}:$ Supp $\mathscr{M} \rightarrow V$ is proper,

(ii) for all $x \in U, \mathscr{M}_{x}$ is a finite $\mathcal{O}_{f(x)}$ module. Then $f_{*}(\mathscr{M})$ is semicoherent over $V$.

THEOREM 4.2. Let $f$ and $\mathscr{M}$ be as above. Suppose $\mathscr{M}^{\prime}$ is a locally finitely generated sheaf on $V$ and $\tau: \mathscr{M}^{\prime} \rightarrow f_{*}(\mathscr{M})$ is a morphism; $\tilde{\tau}: \mathscr{L}^{\prime} \widetilde{\boldsymbol{\otimes}}_{\mathcal{O}_{V}} \mathscr{E}_{V} \rightarrow f_{*}(\mathscr{M}){\widetilde{\boldsymbol{\otimes}_{O_{V}}}}_{\mathscr{E}_{V}}$ is the induced map. Then $\operatorname{Im} \tilde{\tau}(V)$ is closed in $f_{*}(\mathscr{M}) \widetilde{\boldsymbol{\otimes}_{O_{V}}} \mathscr{E}_{V}(V)=f_{*}\left(\mathscr{\mathscr { Q }} \widetilde{\boldsymbol{\otimes}_{\mathcal{O}_{U}}} \mathscr{E}_{U}\right)(V)=$ $\left(\mathscr{A} \widetilde{\otimes}_{O_{U}} \mathscr{E}_{U}\right)(U)$ (all three of which have the same topology).

Now let $f_{0}: \boldsymbol{R}^{n}, 0 \rightarrow \boldsymbol{R}^{p}, 0$ be analytic and finitely $K$-determined. Then there are neighborhoods $U$ and $V$ of 0 in $\boldsymbol{R}^{n}$ and $\boldsymbol{R}^{p}$ and a representative $f: U \rightarrow V$ of $f_{0}$ such that $f$ and $\mathscr{C}=\mathcal{O}_{U}^{p} / d f \mathcal{O}_{U}^{n}$ satisfy the hypotheses of Theorem 4.1. Let $\mathscr{C}^{\prime}=\mathscr{O}_{V}^{p}$ and $\tau: \mathscr{M}^{\prime} \rightarrow f_{*} \mathscr{M}$ be the map induced by $f^{*}: \mathcal{O}_{V}^{p} \rightarrow \mathcal{O}_{I J}^{p}$. Then $\operatorname{Im} \widetilde{\tau}(V)$ is the projection of $f^{*} \mathscr{E}^{p}(V)$ into $\mathscr{E}^{p}(U) / d f^{n} \mathscr{E}^{n}(U)$ (note that $d f^{\mathscr{E}^{n}}(U)$ is closed in $\mathscr{E}^{p}(U)$ by VI. 1.5 of [10]). Theorem 4.2 implies that

$$
d f^{\mathscr{E}}{ }^{n}(U)+f^{*} \mathscr{E}^{p}(V) \text { is closed in } \mathscr{E}^{p}(U) \text {. }
$$

Now assume that $f_{0}$ in addition satisfies condition (3a) (as a germ). Then $f, U$, and $V$ of the above paragraph can be chosen so that, for any finite $S \subset U-\{0\}$, the germ of $f$ at $S$ is stable. Let $\mathscr{E}^{p}(U)_{*}=\left\{g \in \mathscr{E}^{p}(U): g \equiv 0\right.$ in a neighborhood of 0$\}$. Then $\mathscr{E}^{p}(U)_{*} \subset d f_{\mathscr{E}^{n}}(U)+f^{*} \mathscr{E}^{p}(V)$; this is proved by a partition of unity argument just like that on pp. 316-317 of [5]. Clearly $\mathscr{E}^{p}(U)_{*}$ is dense in the set of mappings infinitely flat at 0 . Thus the latter are all in $d f_{\mathscr{E}^{n}}(U)+f^{*} \mathscr{E}^{p}(V)$. Passing to germs, $m_{n}^{\infty} E_{n}^{p} \subset d f_{0} E_{n}^{n}+$ $f_{0}^{*} E_{p}^{p}$.

\section{REFERENCES}

1. G. R. Belickii, Germs of mappings w-determined with respect to a given group, Mat. Sb., 94 (136) (1974), 452-467= Math. USSR.Sb., 23 (1974), 425-440.

2. T. Gaffney, Properties of Finitely Determined Germs, Thesis, Brandeis University, 1975.

3. M. Golubitsky and V. Guillemin, Stable Mappings and Their Singularities, Graduate Texts in Mathematics 14, Springer-Verlag, New York, 1973.

4. J. N. Mather, Stability of $\mathrm{C}^{\infty}$ mappings, III: finitely-determined map germs, Publ. Math. I. H. E. S., 35 (1968), 127-156.

5. - Stability of $C^{\infty}$ mappings, $V$ : transversality, Adv. in Math., 4 (1970), 301336.

6. Infinite dimensional group actions, Asterisque (Soc. Math. France), 32-33 (1976), 165-172. 
7. J. Merrien, Faisceaux analytiques semi-coherents, C. R. Acad. Sci. Paris, 289 (1979), 791-793.

8. - Applications des faisceaux analytiques semi-coherent aux fonctions differentiables, C. R. Acad. Sci. Paris, 290 (1980), 37-39.

9. - Faisceaux analytiques semi-coherents et fonctions differentiables, These, Universite de Rennes, 1980.

10. J. C. Tougeron, Ideaux de Fonctions Differentiables, Ergebnisse Band 71, SpringerVerlag, New York, 1972.

11. L. C. Wilson, Infinitely determined mapgerms, Canad. J. Math., to appear.

12. — A-codimension of mappings, in preparation.

Received October, 1979 and in revised form January 9, 1981.

UNIVERSITY OF HAWAII

Honolulu, HI 96822 



\section{PACIFIC JOURNAL OF MATHEMATICS}

\section{EDITORS}

DONALD BABBITT (Managing Editor)

University of California

Los Angeles, California 90024

HUGO ROSSI

University of Utah

Salt Lake City, UT 84112

C. C. MOORE and ARTHur AGUS

University of California

Berkeley, CA 94720
J. DUGUNDJI

Department of Mathematics

University of Southern California

Los Angeles, California 90007

R. FINN and J. MILGRAM

Stanford University

Stanford, California 94305

\section{ASSOCIATE EDITORS}
R. Arnes
E. F. BECKENBACH
B. H. NeumanN
F. WOLF
K. YösHIDA

\section{SUPPORTING INSTITUTIONS}

UNIVERSITY OF ARIZONA

UNIVERSITY OF BRITISH COLUMBIA

CALIFORNIA INSTITUTE OF TECHNOLOGY

UNIVERSITY OF CALIFORNIA

MONTANA STATE UNIVERSITY

UNIVERSITY OF NEVADA, RENO

NEW MEXICO STATE UNIVERSITY

OREGON STATE UNIVERSITY
UNIVERSITY OF OREGON

UNIVERSITY OF SOUTHERN CALIFORNIA

STANFORD UNIVERSITY

UNIVERSITY OF HAWAII

UNIVERSITY OF TOKYO

UNIVERSITY OF UTAH

WASHINGTON STATE UNIVERSITY

UNIVERSITY OF WASHINGTON 


\section{Pacific Journal of Mathematics}

Vol. 102, No. $1 \quad$ January, 1982

S. Agou, Degré minimum des polynômes $f\left(\sum_{i=0}^{m} a_{i} X^{p^{r i}}\right)$ sur les corps finis

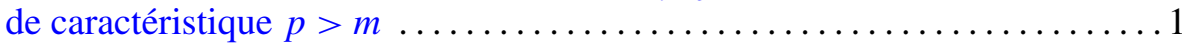

Chi Cheng Chen, On the image of the generalized Gauss map of a complete

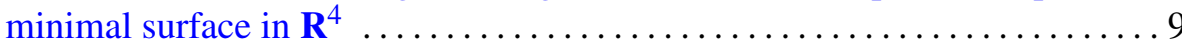

Thomas Curtis Craven and George Leslie Csordas, On the number of real

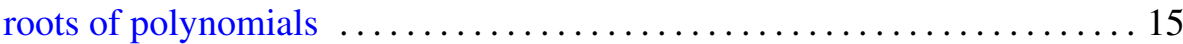

Allan L. Edelson and Kurt Kreith, Nonlinear relationships between oscillation and asymptotic behavior ....................... 29

B. Felzenszwalb and Antonio Giambruno, A commutativity theorem for

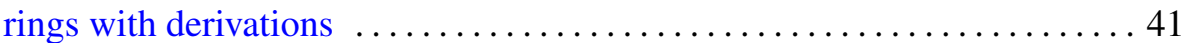

Richard Elam Heisey, Manifolds modelled on the direct limit of lines . . . . . 47

Steve J. Kaplan, Twisting to algebraically slice knots $\ldots \ldots \ldots \ldots \ldots \ldots 5$

Jeffrey C. Lagarias, Best simultaneous Diophantine approximations. II.

Behavior of consecutive best approximations $\ldots \ldots \ldots \ldots \ldots \ldots \ldots 61$

Masahiko Miyamoto, An affirmative answer to Glauberman's conjecture . . . 889

Thomas Bourque Muenzenberger, Raymond Earl Smithson and L. E.

Ward, Characterizations of arboroids and dendritic spaces ........... 107

William Leslie Pardon, The exact sequence of a localization for Witt

groups. II. Numerical invariants of odd-dimensional surgery

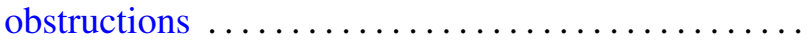

Bruce Eli Sagan, Bijective proofs of certain vector partition identities

Kichi-Suke Saito, Automorphisms and nonselfadjoint crossed products ...

John Joseph Sarraille, Module finiteness of low-dimensional PI rings ...

Gary Roy Spoar, Differentiable curves of cyclic order four . .

William Charles Waterhouse, Automorphisms of quotients of $\Pi \mathrm{GL}\left(n_{i}\right)$

Leslie Wilson, Mapgerms infinitely determined with respect to right-left equivalence

Rahman Mahmoud Younis, Interpolation in strongly logmodular

algebras 